\title{
Confirmación diagnóstica de alergia a la amoxicilina en niños tratados en una sección de alergia pediátrica
}

Diagnostic confirmation of amoxicillin allergy in children treated at a division of Pediatric Allergy

\author{
Dr. Christian Morelo Torres ${ }^{a}$ Dr. Alfredo Eymann ${ }^{b}$, Dra. Natalia Petriz ${ }^{a}$ y Dr. Claudio A. Parisi ${ }^{a}$
}

\section{RESUMEN}

La amoxicilina es un antibiótico betalactámico comúnmente indicado en pediatría y es la causa más frecuente de alergia a medicamentos.

Objetivos. Determinar la proporción de alergia confirmada a amoxicilina en niños con sospecha diagnóstica, atendidos en una sección de alergia pediátrica.

Población y métodos. Estudio descriptivo retrospectivo entre enero de 2009 y enero de 2017, en menores de 18 años con sospecha diagnóstica de alergia a amoxicilina. Se realizó el diagnóstico según interrogatorio y pruebas específicas.

Resultados. Fueron incluidos 234 pacientes; se diagnosticó alergia a la amoxicilina en el 10,7 \% (intervalo de confianza del $95 \%$ : 7-15). Estos pacientes tenían mayor prevalencia de síntomas inmediatos (el $40 \%$ vs. el $22 \%, \mathrm{p}=0,048)$ y de exposición previa a betalactámicos (el $84 \%$ vs. el $56 \%$, p =0,007). Conclusión. La confirmación de alergia a la amoxicilina en niños derivados a especialistas fue del 10,7 \%.

Palabras clave: hipersensibilidad, fármacos, betalactámicos, niños, epidemiología.

http: / / dx.doi.org/10.5546/ aap.2020.47

Texto completo en inglés:

http: / / dx.doi.org/10.5546/ aap.2020.eng.47

Cómo citar: Morelo Torres C, Eymann A, Petriz N, Parisi CA. Confirmación diagnóstica de alergia a la amoxicilina en niños tratados en una sección de alergia pediátrica. Arch Argent Pediatr 2020;118(1):47-51. a. Sección Alergia Pediátrica del Hospital Italiano de Buenos Aires.

b. Servicio de Clínica Pediátrica del Hospital Italiano de Buenos Aires.

Correspondencia:

Dr. Claudio A. Parisi: claudio.parisi@hiba.org.ar

Financiamiento: Ninguno.

Conflicto de intereses: Ninguno que declarar.

Recibido: 28-2-2019

Aceptado: 17-6-2019

\section{INTRODUCCIÓN}

Las reacciones alérgicas a medicamentos (RAM) se clasifican, según la rapidez de aparición de los síntomas, en inmediatas y no inmediatas. ${ }^{1,2}$ Las primeras pueden manifestarse desde síntomas limitados a la piel hasta reacciones graves y potencialmente fatales. ${ }^{2}$ Las segundas ocurren después de una hora de haber ingerido el antibiótico; la gran mayoría son leves; incluyen exantemas maculopapulares. ${ }^{2,3} \mathrm{y}$, raramente, pueden presentarse con cuadros más graves. ${ }^{1}$

La amoxicilina es la causa más habitual de RAM. ${ }^{4}$ Alrededor del $70 \%$ de los pacientes que padecen infecciones virales son medicados de forma empírica con este antibiótico y los exantemas maculopapulares frecuentemente secundarios a estos cuadros son, a menudo, interpretados de modo erróneo como RAM.,5

A pesar de esto, la mayoría de los pacientes no suelen confirmar el diagnóstico. Según nuestro conocimiento, existen escasos datos epidemiológicos de esta enfermedad en niños argentinos. El objetivo de este trabajo fue determinar la proporción de alergia confirmada a amoxicilina en niños con sospecha diagnóstica atendidos en una sección de alergia pediátrica.

\section{POBLACIÓN Y MÉTODOS}

Se realizó un estudio descriptivo retrospectivo entre el 1 de enero de 2008 y el 1 enero de 2017.

La población fueron los pacientes menores de 18 años que tuvieran definido como problema en la historia clínica electrónica (HCE) alergia a amoxicilina, sospecha de alergia a amoxicilina, alergia a betalactámicos o sospecha de alergia a betalactámicos y que hubieran realizado, al menos, una consulta en la Sección de Alergia Pediátrica.

Se definió como criterio de exclusión a los pacientes con información incompleta, alergia a otro betalactámico, a aquellos que no realizaron las pruebas diagnósticas específicas y pacientes con reacciones no inmediatas graves, como síndrome de Stevens-Johnson, necrólisis 
epidérmica tóxica y reacción cutánea por fármacos con eosinofilia y síntomas sistémicos. ${ }^{1}$

Se analizaron las siguientes variables: edad, sexo, antecedentes personales y familiares en primer grado de alergia (asma, rinitis alérgica, dermatitis atópica, alergia a alimentos, alergia a fármacos y/o alergia a picadura de insectos), exposición previa a la amoxicilina y / o antibióticos betalactámicos.

Se realizaron las siguientes pruebas diagnósticas:

Dosaje de inmunoglobulina $E$ ( $I g E$ ) específica sérica: Para amoxicilina, penicilina $\mathrm{G}$ y V, y ampicilina mediante ensayo por inmunoabsorción ligado a enzimas (enzyme-linked immunosorbent assay; ELISA, por sus siglas en inglés). Se consideraron positivos los valores mayores de $0,36 \mathrm{UI} / \mathrm{ml}$.

Prueba cutánea de lectura inmediata (PCLI): ${ }^{6}$ Se utilizó amoxicilina (concentración: $25 \mathrm{mg} / \mathrm{ml}$ ). ${ }^{6} \mathrm{Se}$ consideró positivo el habón mayor de $3 \mathrm{~mm}$ con respecto al testigo negativo.

Intradermorreacción: Técnica de Mantoux, en que se utilizó amoxicilina en concentración de $25 \mathrm{mg} / \mathrm{ml} .{ }^{6}$ Se consideró positiva la presencia de un habón con eritema, mayor o igual a $3 \mathrm{~mm}$ de diámetro con respecto al testigo negativo. ${ }^{6} \mathrm{Al}$ no contar, en nuestro país, con los determinantes mayores, bencilpeniciloil-polilisina y la mezcla de determinantes menores ${ }^{4}$ estos no fueron testeados.

Prueba de parche atópico (PPA): Se colocó la dilución pura del antibiótico ${ }^{7}$ bajo oclusión en placas de Finn en el área interescapular durante 2 días y se realizaron lecturas a las 48 y a las $96 \mathrm{~h}$. Se consideraron pruebas positivas de acuerdo con las graduaciones del Grupo Internacional de Investigación en Dermatitis de Contacto. ${ }^{8}$

Prueba de provocación controlada (PPC): Se administró el antibiótico por vía oral en dosis terapéuticas de $80 \mathrm{mg} / \mathrm{kg} /$ día, en el ambiente intrahospitalario, en tres tomas fraccionadas con intervalos de 20 minutos. Aquellos que, durante la PPC, tuvieron síntomas de hipersensibilidad inmediata, test positivo, se consideraron alérgicos. Aquellos que no presentaron síntomas sospechosos continuaron tomando el antibiótico durante 5 días y quedaron en contacto ambulatorio con el equipo médico para reportar eventuales síntomas no inmediatos, protocolo modificado de Mori et al. ${ }^{3}$

Se definió alergia a la amoxicilina la presencia

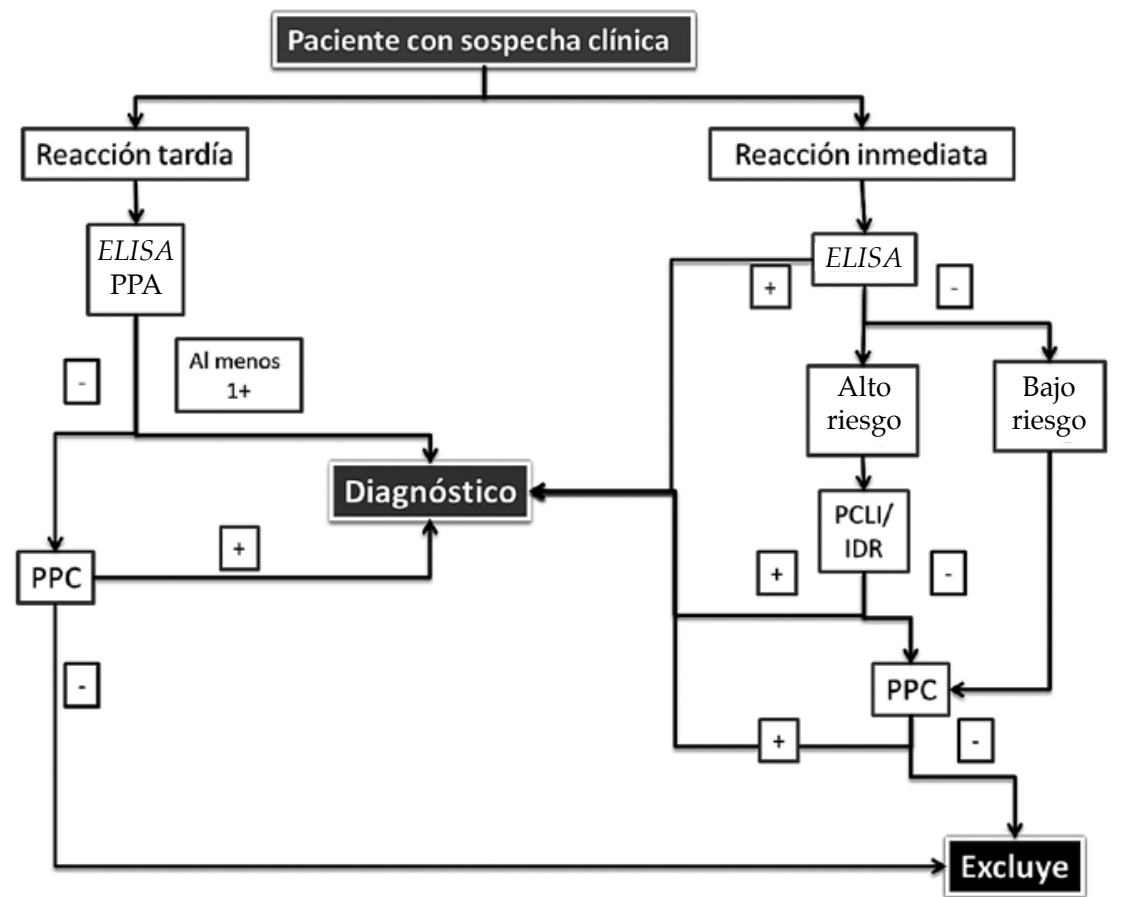

PPA: prueba de parche atópico; PPC: prueba de provocación controlada; PCLI: prueba cutánea de lectura inmediata; IDR: intradermorreacción. 
de síntomas inmediatos (anafilaxia, angioedema, urticaria, broncoespasmo) y/o la presencia de síntomas no inmediatos (exantemas, eccemas maculopapulosos, pustulosos, bullosos $)^{9}$ con, al menos, un test confirmatorio positivo.

Se consideraron pacientes con alto riesgo de alergia los mayores de 12 años y/o con historia de episodios graves (consulta a la central de emergencias, síntomas de dificultad respiratoria y/o uso de adrenalina). ${ }^{10}$ A estos pacientes, si el resultado del ELISA fue negativo, se les realizaron las PCLI e intradermorreacción; a los pacientes considerados de bajo riesgo se les realizó directamente el test de provocación después del ELISA negativo.

Se realizó PPA a los pacientes con reacciones tardías. A todos los pacientes, independientemente del mecanismo involucrado, se les realizó el ELISA. Se describe el proceso diagnóstico en la Figura 1.

Este estudio fue aprobado por el Comité de Ética y Protocolos de Investigación de la Institución.

\section{Análisis estadístico}

Se presentaron las variables cuantitativas como media y desvío estándar (DE), y las variables categóricas, como frecuencias y porcentajes. Se calculó la proporción de alergia confirmada a la amoxicilina, tomando como numerador el número de pacientes con diagnóstico confirmado de alergia a amoxicilina y, como denominador, el número total de pacientes en consulta ambulatoria que fueron derivados o concurrieron espontáneamente a la Sección de Alergia Pediátrica con sospecha de alergia a la amoxicilina. Las diferencias entre variables continuas se analizaron mediante el $t$-test para muestras independientes, y las proporciones entre variables categóricas, mediante la prueba de $c h i^{2}$. Se realizó un modelo multivariado con las variables estadísticamente significativas en el análisis univariado. Se consideró significativo un valor de $p<0,05$. Se utilizó el programa estadístico STATA versión 13.

\section{RESULTADOS}

Ingresaron al estudio un total de 234 pacientes (Figura 2). Fueron varones el $52 \%$, con una media de edad de 3,6 años (DE 3,1 años). Del total de los pacientes, 178 (el $76 \%$ ) presentaron síntomas retardados, y 56 (el $24 \%$ ) presentaron síntomas inmediatos (Tabla 1); 138 (el 59 \%) habían estado previamente expuestos a algún antibiótico betalactámico, por lo menos, en una ocasión.

Fueron diagnosticados como alérgicos a la amoxicilina 25 pacientes, que representaron el

FIGURA 2. Diagrama de flujo de pacientes incluidos en el estudio

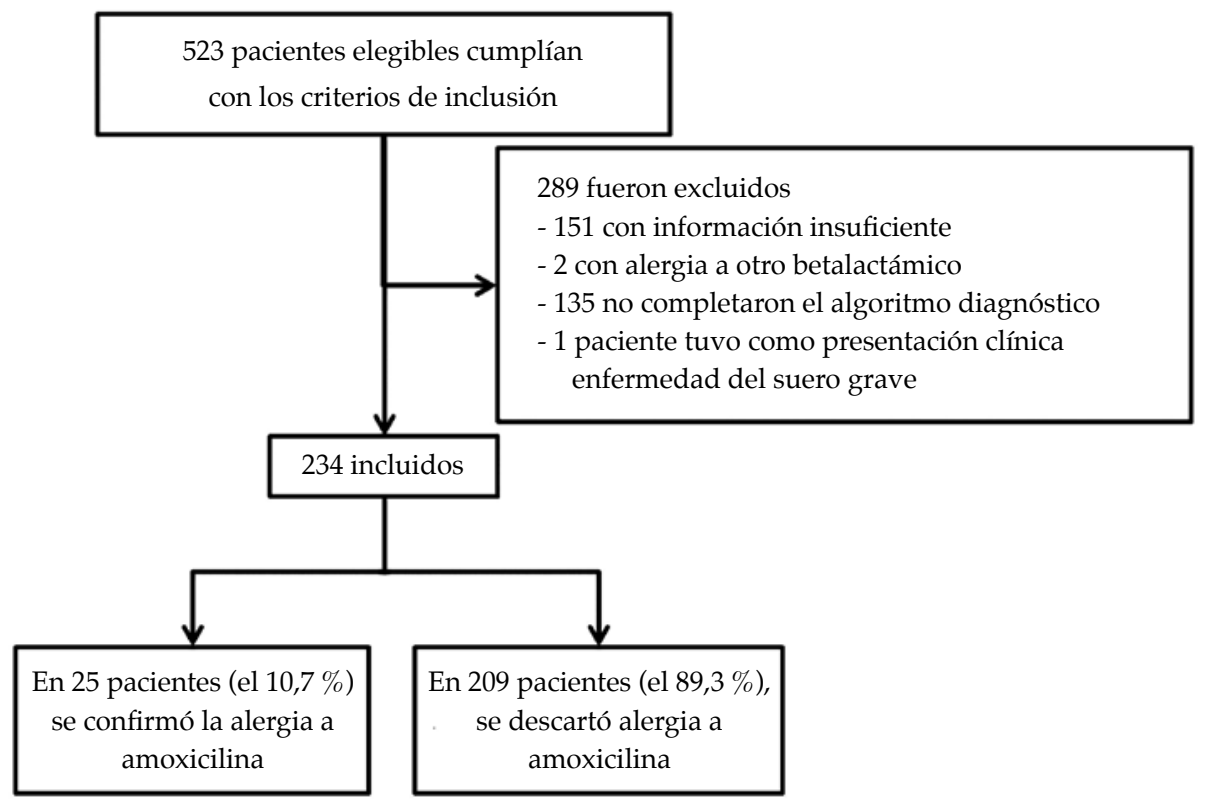


10,7 \% (intervalo de confianza -IC- del $95 \%$ : 7-15) de la población de estudio (Figura 2). El diagnóstico se confirmó mediante ELISA, pruebas cutáneas o PPC en 9 (el $36 \%), 3$ (el $12 \%$ ) y 13 (el $52 \%$ ) de los niños, respectivamente.

En el análisis univariado, los pacientes con alergia a la amoxicilina confirmada tenían mayor prevalencia de síntomas inmediatos (el $40 \%$ vs. el $22 \%, \mathrm{p}=0,048)$ y de exposición previa a betalactámicos (el $84 \%$ vs. el $56 \%$, p =0,007). No se encontraron diferencias en otras variables (Tabla 2).
En el análisis multivariado, la presencia de síntomas inmediatos y la exposición previa a betalactámicos mantuvieron la significancia estadística. Los niños que se presentaron con síntomas inmediatos tuvieron un odds ratio (OR) para alergia confirmada a amoxicilina de 2,45 (IC $95 \%$ : 1,01-5,87; $\mathrm{p}=0,049$ ). A su vez, los niños con antecedentes de consumo de betalactámicos tuvieron un $O R$ para alergia confirmada a la amoxicilina de 4,24 (IC $95 \%$ : 1,39-12,90; $\mathrm{p}=0,011$ ).

TABLA 1. Características clínica de los pacientes con sospecha de alergia a la amoxicilina $n=234$

\begin{tabular}{|c|c|c|c|c|}
\hline & & & $\mathbf{n}$ & $\%$ \\
\hline \multirow[t]{2}{*}{ Sexo } & Mujer & & 114 & 48,7 \\
\hline & Varón & & 120 & 51,3 \\
\hline \multirow[t]{6}{*}{ Tipo de síntomas } & Inmediatos & Cutáneos (urticaria/angioedemia, & & \\
\hline & & exantema maculopapular) & 52 & 22,2 \\
\hline & & Digestivos & 1 & 0,4 \\
\hline & & Anafilaxia & 3 & 1,3 \\
\hline & Retardados & Exantema máculopapular & 128 & 54,7 \\
\hline & & Urticaria & 50 & 21,4 \\
\hline \multirow[t]{2}{*}{ Exposición previa a betalactámicos } & Sí & & 138 & 59 \\
\hline & No & & 96 & 41 \\
\hline \multirow[t]{5}{*}{ Antecedentes perosnales de alergia } & Sí & Asma-broncoespasmos & 37 & 50,7 \\
\hline & & Rinitis & 30 & 41 \\
\hline & & Dermatitis atópica & 9 & 12,3 \\
\hline & & Otras & 10 & 14 \\
\hline & No & & 161 & 69 \\
\hline \multirow[t]{2}{*}{ Antecedentes familiares de alergia } & Sí & & 112 & 48 \\
\hline & No & & 112 & 52 \\
\hline
\end{tabular}

TABLA 2. Comparación de los pacientes con alergia a la amoxicilina

\begin{tabular}{|c|c|c|c|c|}
\hline & & $\begin{array}{c}\text { Sin alergia } \\
(\mathbf{n}=209) \\
\% \\
\end{array}$ & $\begin{array}{c}\text { Con alergia } \\
(\mathrm{n}=25) \\
\% \\
\end{array}$ & ${ }^{*}$ Valor de $p$ \\
\hline * Sexo & $\begin{array}{l}\text { Mujer } \\
\text { Varón }\end{array}$ & $\begin{array}{l}48 \\
52\end{array}$ & $\begin{array}{l}52 \\
48\end{array}$ & 0,711 \\
\hline${ }^{* *}$ Edad en años & (media $\pm \mathrm{DE})$ & $3,48 \pm 3,20$ & $3,92 \pm 2,94$ & 0,495 \\
\hline * Síntomas inmediatos & $\begin{array}{l}\text { Sí } \\
\text { No }\end{array}$ & $\begin{array}{l}22 \\
88\end{array}$ & $\begin{array}{l}40 \\
60\end{array}$ & $\begin{array}{l}0,048 \\
0,048\end{array}$ \\
\hline * Exposición previa a betalactámicos & $\begin{array}{l}\text { Sí } \\
\text { No }\end{array}$ & $\begin{array}{l}56 \\
44\end{array}$ & $\begin{array}{l}84 \\
26\end{array}$ & 0,007 \\
\hline * Antecedentes personales de aenfermedad alérgica & $\begin{array}{l}\text { Sí } \\
\text { No }\end{array}$ & $\begin{array}{l}30 \\
70\end{array}$ & $\begin{array}{l}44 \\
66\end{array}$ & 0,148 \\
\hline${ }^{*}$ Antecedentes familiares de enfermedad alérgica & $\begin{array}{l}\text { Sí } \\
\text { No }\end{array}$ & $\begin{array}{l}45 \\
55\end{array}$ & $\begin{array}{l}60 \\
40\end{array}$ & 0,206 \\
\hline
\end{tabular}

* prueba de chi².

** prueba $t$ de Student.

DE: desvío estándar. 


\section{DISCUSIÓN}

Si bien existe una alta sospecha de alergia a este antibiótico, un alto porcentaje de los niños estudiados no son alérgicos. ${ }^{11,12}$ En este estudio, la confirmación de alergia a la amoxicilina en niños derivados a especialistas fue del $10,7 \%$, similar a lo reportado por Mori et al., ${ }^{3}$ quienes identificaron como alérgicos al 9,6\% entre 200 niños italianos utilizando una metodología de estudio similar.

Los niños que presentaron síntomas inmediatos tuvieron 1,5 veces más chances de presentar una RAM que aquellos que tuvieron síntomas retardados, y aquellos previamente expuestos a antibióticos betalactámicos tuvieron una fuerte asociación con la confirmación del diagnóstico de alergia a amoxicilina con una chance 3,2 veces mayor.

No se encontró una relación significativa entre los antecedentes personales y familiares de alergia, al igual que el estudio de Ponvert y col., ${ }^{13}$ y a diferencia del de Faitelson y col. ${ }^{10}$

Existen pocos estudios de confirmación diagnóstica y, en general, un sobrediagnóstico de esta afección. ${ }^{14}$ Este trabajo aborda un problema para el cual no hay suficientes datos y subraya la importancia del interrogatorio y las pruebas diagnósticas para evitar el impacto negativo que genera categorizar a los pacientes con una afección inexistente.

El estudio presenta algunas limitaciones: se utilizó un diseño retrospectivo; del total de la muestra, el 44,7\% cumplió con los criterios de inclusión; se realizó en un solo centro hospitalario y se incluyeron niños derivados a especialistas, por lo que se puede inferir que la prevalencia en la población pediátrica general podría ser menor.

En conclusión, en nuestro medio, más de la mitad de los niños que consultan al especialista por sospecha de alergia a la amoxicilina no poseen tal afección.

\section{Agradecimiento}

A la Dra. Carolina Antonietti por su importante colaboración en este trabajo.

\section{REFERENCIAS}

1. Romano A, Warrington R. Antibiotic allergy. Immunol Allergy Clin North Am. 2014; 34(3):489-506.

2. Pavlos R, Mallal S, Ostrov D, Buus S, et al. T Cell-mediated hypersensitivity reactions to drugs. Annu Rev Med. 2015; 66:439-54.

3. Mori F, Cianferoni A, Barni S, Pucci N, et al. Amoxicillin Allergy in children: five-day provocation test in the diagnosis of nonimmediate reactions. J Allergy Clin Immunol Pract. 2015; 3(3):375-80.e1.

4. Comité Nacional de Alergia. Reacciones alérgicas a betalactámicos en pediatría: recomendaciones para su diagnóstico y tratamiento. Arch Argent Pediatr. 2019; 117(Suppl 1):S24-36.

5. Jappe U. Amoxicillin-induced exanthema in patients with infectious mononucleosis: allergy or transient immunostimulation? Allergy. 2007; 62(12):1474-5.

6. Torres MJ, Blanca M, FernándezJ, Romano A, etal. Diagnosis of immediate allergic reactions to beta-lactam antibiotics. Allergy. 2003; 58(10):961-72.

7. DeGroot AC. Patch Testing. Test concentration and vehicles for 3700 chemicals. 2nd ed. New York: Elsevier; 1994.

8. Fischer T, Maibach HI. Patch Testing in allergic contact dermatitis. Sem Dermatol. 1986; 5:59-68.

9. Demoly P, Romano A. Update on Beta-lactam Allergy diagnosis. Curr Allergy Asthma Rep. 2005; 5(1):9-14.

10. Faitelson Y, Boaz M, Dalal I. Asthma, Family History of Drug Allergy, and Age Predict Amoxicillin Allergy in Children. J Allergy Clin Immunol Pract. 2018; 6(4):1363-7.

11. Impicciatore P, Choonara I, Clarkson A, Provasi D, et al. Incidence of adverse drug reactions in paediatric in/ out-patients: a systematic review and meta-analysis of prospective studies. Br J Clin Pharmacol. 2001; 52(1):77-83.

12. Romano A. Recognising antibacterial hypersensitivity in children. Paediatr Drugs. 2000; 2(2):101-12.

13. Ponvert C, Perrin $Y$, Bados-Albiero A, Le Bourgeois M, et al. Allergy to betalactam antibiotics in children: results of a 20-year study based on clinical history, skin and challenge tests. Pediatr Allergy Immunol. 2011; 22(4):411-8.

14. Erkoçoglu M, Kaya A, Civelek E, Ozcan C, et al. Prevalence of confirmed immediate type drug hypersensitivity reactions among school children. Pediatr Allergy Immunol. 2013; 24(2):160-7. 